${ }^{1}$ Zentrum für Ausbildung im Gesundheitswesen Kanton Zürich, Schweiz

${ }^{2}$ Fachstelle für Entwicklung und Forschung, Pflegezentren der Stadt Zürich, Schweiz

${ }^{3}$ Universität Zürich, Zentrum für Gerontologie, Schweiz

${ }^{4}$ UMIT Private Universität für Gesundheitswissenschaften, Medizinische Informatik und Technik, Hall in Tirol, Österreich Daniela Händler-Schuster (Mag.) ${ }^{1}$, Heike Geschwindner (MHSc)², Sandra Oppikofer (Dr. phil.)³, Christa Them (Univ.-Prof. Dr.) $)^{4}$

\title{
Wie erleben Erfasserinnen den Umgang mit der Observed Emotional Rating Scale?
}

- Was ist (zu dieser Thematik) schon bekannt?

Die Observed Emotion Rating Scale ermöglicht es, Emotionen bei Menschen mit kognitiven Beeinträchtigungen zu messen.

- Was ist neu? Anwenderinnen beschreiben ihre Erfahrungen mit der Observed Emotion Rating Scale nach der gezielten Anwendung in der Praxis.

- Welche Konsequenzen haben die Ergebnisse für die Pflegepraxis? Die Anwendung der Observed Emotion Rating Scale sollte im Rahmen einer Anwenderschulung gut vorbereitet werden. Um valide Ergebnisse zu erzielen, sind pflegerische Kenntnisse Voraussetzung für die Anwendung.

Da im deutschsprachigen Raum bisher kaum empirische Daten zur Anwendung der Observed Emotion Rating Scale (OERS) bei demenzerkrankten Personen vorliegen, sollte im Rahmen des Pilotprojekts "Agitation" untersucht werden, wie Erfasserinnen den Einsatz des Instruments OERS erleben. Zu diesem Zweck konnten 12 Probandinnen gewonnen werden, die in drei Pflegezentren im Kanton Zürich Beobachtungen mit dem Instrument OERS durchführten. Der überwiegende Teil der Probandinnen verfügte über pflegerische Qualifikationen bzw. Erfahrung im Umgang mit demenzerkrankten Personen. Die Datensammlung erfolgte anhand von drei Fokusgruppeninterviews, die mittels einer qualitativen Inhaltsanalyse ausgewertet worden waren. Die vorläufigen Ergebnisse aus den Fokusgrup-

Manuskript erstmals eingereicht am 9.1.2009 Endgültige Fassung eingereicht am 16.6.2009 peninterviews wurden von denselben Probandinnen anhand eines Fragebogens validiert. Die Kategorien "fehlende Eindeutigkeit", "fehlende Kodiermöglichkeit", "Demenzschwere", "Einschätzen von Emotionen", "Einflussfaktoren" und "Anwenderschulung" zeigten, dass das Wissen um die Demenzerkrankung genauso bedeutsam ist wie eine geübte Handhabung

\section{Einleitung}

Einhergehend mit den demografischen Veränderungen in der Bevölkerung wächst auch die Zahl der Demenzerkrankten. Die Hochrechnung altersspezifischer Inzidenzraten deutet darauf hin, dass in der Schweiz jährlich um die 18000 ältere Personen neu an hirnorganischen Störungen erkranken Damit ist die Demenz eine der häufigsten und folgenreichsten neurodegenerativen Erkrankungen im höheren Alter und stellt die Gesellschaft vor eine große Herausforderung (Höpflinder OERS. ger \& Hugentobler, 2004; Weyerer, 2005).

Wenn der überwiegend verbale Weg der Kommunikation zwischen demenzerkrankten Personen und ihrem sozialen Umfeld nicht mehr möglich ist, bekommt vor allem das Erkennen von Emotionen eine besondere Bedeutung, weil auch Wünsche und Ansprüche nonverbal von demenzerkrankten Personen geäußert werden. Pflegende können in der zwischenmenschlichen Kommunikation individuelle Bedürfnisse erspüren und diese in der Interaktion mit Demenzerkrankten berücksichtigen.

Häufig kann schon allein die Anwesenheit von Personen im näheren Umfeld Sicherheit und Orientierung vermitteln, womit Betroffene sogar im späten Stadium ihrer Erkrankung auch emotional erreicht werden können (Gonzalez-Salvador, Lyketsos, Baker, Hovanec, Roques, Brandt et al., 2000). Eine Möglichkeit, um Emotionen zu erfassen, ist die quantifizierende Methode mittels Observed Emotion Rating Scale (OERS, synonym: Apparent Affect Rating Scale), die von Lawton, Van Haitsma \& Klapper (1996a) entwickelt wurde. Das Ziel der OERS ist es, Menschen mit kognitiven Beeinträchtigungen besser zu verstehen und mit der Einschätzung von Emotionen auf individuelle Bedürfnisse reagieren zu können. Die Ergebnisse aus den Untersuchungen von Lawton et al. (1996a) zeigten, dass sich die Einschätzungsskala OERS dazu eignet, Emotionen bei Menschen sowohl mit einem leichten als auch sehr schweren Grad der Demenzerkrankung zu erfassen (Lawton et al., 1996a).

\section{Einbettung der Studie}

Das Zentrum für Gerontologie in Zürich (ZfG) hat im Jahr 2006 ein Projekt 
zur Entwicklung und Erprobung einer Instrumentenbatterie zur Erhebung der Lebensqualität von Menschen mit einer Demenz lanciert (ILQ) und im Frühjahr 2007 einige Instrumente in einer Pilotstudie getestet. Die vorliegende Untersuchung stellte eine Ergänzung zum Pilotprojekt dar, wodurch die Anwendung der OERS fokussiert wurde.

\section{Methodisches Vorgehen}

\section{Anwenderschulung}

Für die Vorbereitung auf die Einschätzungen mittels OERS fand eine eintägige Anwenderschulung durch die Projektleitung mit allen Teilnehmenden im Gesamtprojekt (ILQ) statt. Die Schulung umfasste neben der Einführung in das ZfG Pilotprojekt «Agitation», Informationen zum Zeitplan und zum Vorgehen bei der Datenerhebung sowie ein Referat zur Demenzerkrankung. Die eigentliche Anwendung, und das Erkennen und Einschätzen von Emotionen mit dem Instrument OERS, wurde anhand von Filmsequenzen aus dem Demenzpflegealltag im Kanton Zürich und dem zu diesem Zweck Deutsch vertonten original Schulungsfilm von Lawton et al. (Philadelphia Geriatric Center, 1996) geübt und reflektiert. Mit der gemeinsamen Kodierung der Sequenzen sollte eine möglichst hohe Interrater-Reliabilität erzielt werden.

\section{Die Observed Emotional Rating Scale}

Das englische Originalinstrument OERS wurde vom ZfG durch einen professionellen Übersetzer ins Deutsche übersetzt. Eine Rückübersetzung vom Deutschen zum Englischen lag vom Institut für Gerontologie in Heidelberg vor. Weil diese in fast allen Punkten mit der Originalversion übereinstimmte (Lawton et al., 1996b), wurde von einer neuerlichen Rückübersetzung des Instrumentes abgesehen. Für das Pilotprojekt «Agitation» passte Oppikofer (2008) lediglich das Layout der OERS an. Das Datenblatt enthielt in Anlehnung an Lawton et al. (1996b) fünf Gefühlszustände; zwei positive: «Freude» und «allgemeine Aufmerksamkeit/Interesse» und drei negative: «Traurigkeit», «Angst» und «Ärger/ Wut». Jede Einschätzung eines Bewohners mit der OERS wurde anhand einer fünfminütigen Beobachtungsphase durchgeführt. Alle in diesem Zeitraum auftretenden Emotionen wurden anschließend auf dem Datenblatt festgehalten.

\section{Fragestellungen}

Die hier vorliegende Forschungsarbeit ging den folgenden Fragen nach:

- Wie erleben Erfasserinnen den Einsatz mit dem Instrument OERS?

- Welche Inhalte lassen sich aus den Erfahrungen der Erfasserinnen für eine Schulung ableiten?

Der vorliegende Artikel konzentriert sich ausschließlich auf das Erleben von Erfasserinnen während der Beobachtung und Einschätzung mit der OERS. Mit den Ergebnissen sollten Empfehlungen für den Umgang mit der Einschätzungsskala OERS formuliert werden. Es wurde für diesen Zweck folgende Fragestellung formuliert: Wie erleben Erfasserinnen die Anwendung der OERS bei Menschen mit einer Demenz im stationären Wohnumfeld?

\section{Material und Methode}

Zur Beantwortung der Forschungsfrage wurde ein qualitativer Forschungsansatz gewählt, der die Erfahrungen der Probandinnen ins Zentrum stellt (Flick, 2006).

Für die Untersuchung wurden die vier Prinzipien qualitativer Forschung - Offenheit, Kommunikation, Naturalistizität und Interpretativität - beachtet (Lamnek, 2005). Mit der Offenheit und der Kommunikation versuchte die Erstautorin eine Grundhaltung gegenüber den Untersuchungspersonen und der Methode einzunehmen um damit der Realitätsnähe mehr Gewicht zu geben als der Theorie. Ebenso wurde eine bewusste Offenheit und Flexibilität innerhalb der Interviews angestrebt, indem die Erstautorin sich den Probandinnen im Forschungsprozess im Tempo der jeweiligen Erzählweisen angepasst hat. Es wurde im gesamten Untersuchungsverlauf eine natürliche Atmosphäre angestrebt. Mit der Naturalistizität und der Interpretativität wurde versucht, möglichst natürliche Situationen $\mathrm{zu}$ schaffen und soziales Handeln realitätsnah zu erforschen. Beobachtungen gewinnen in den letzten Jahren neben anderen forschungsgeleiteten Überlegungen zunehmend an Bedeutung und bedürfen einer nachträglichen Reflexion der eigenen Rolle im Feld. Situationsbeobachtungen erfordern ein Sich-Einstellen auf die Beobachtungssituation, um das Forschungsfeld nicht unnötig zu beeinflussen (Scheffer, 2002).

\section{Datenerhebung}

Für die Datenerhebung wurde die Methode des halbstrukturierten Interviews in Fokusgruppen gewählt (Flick, 2006). Jede Fokusgruppe bestand aus vier Erfasserinnen, die gemeinsam in einer Einrichtung Beobachtungen mithilfe der OERS durchgeführt hatten. In der Forschung werden Fokusgruppeninterviews verwendet, um das Verstehen gezielt zu generieren, Meinungen zu erforschen und Erfahrungen zu beschreiben. Fokusgruppen eignen sich zur Orientierung im Feld und zur Exploration von neuem Material (Kean, 2000; Flick, 2006). In der vorliegenden Untersuchung sollten durch das erzählende Beschreiben der Anwendung der OERS gezielt die gemachten Erfahrungen generiert werden. Um eine einheitliche Vorgehensweise zu erreichen, wurde ein Interviewleitfaden entwickelt. Dieser orientierte sich an Fragen, 
die das zentrale Erleben der Probandinnen in das Zentrum der Interviews stellen sollte, z.B.: Wie ging es Ihnen während der Einschätzung der Emotionen? Welche Situationen in der Beobachtung waren für Sie schwierig zu erfassen?

Alle Interviews wurden auf einem digitalen Diktier- und Aufnahmegerät aufgezeichnet und anschließend vom Schweizerdeutschen ins Standarddeutsche transkribiert. Alle Probandinnen wurden darüber informiert, dass die Forscherin während des Gespräches keine Kommentare über das Gesprochene abgeben und keine Fragen beantworten würde. Jede Person hatte die Möglichkeit, im Anschluss an die Interviews Fragen zu stellen oder zu diskutieren.

\section{Datenanalyse}

Die Analyse der Daten erfolgte nach dem Ansatz der qualitativen Inhaltsanalyse nach Mayring (2003). Zentrale Aussagen der Probandinnen sollten durch die Abstraktion auf Kategorien reduziert werden und gleichzeitig sollten die wesentlichen Inhalte aus dem Grundmaterial erhalten bleiben (Flick, 2006; Häder, 2006).

Im Anschluss an die Kategorienbildung wurde einen Fragenbogen entwickelt, in dem wesentliche Aussagen aus den gebildeten Kategorien als Items wieder aufgenommen worden waren. Der Fragebogen umfasste zu jeder Kategorie drei bis sieben Aussagen, welche anhand einer Likertskala mit sechs Antwortmöglichkeiten («stimme völlig zu» bis «stimme absolut nicht zu») beurteilt worden waren. Ziel des Fragebogens war, die Aussagen aus den Interviews von den Probandinnen verifizieren bzw. falsifizieren zu lassen, um so den Ergebnissen aus der qualitativen Analyse mehr Gewicht geben zu können.

Aufgrund der kleinen Stichprobe wurde kein Prätest durchgeführt. Für die Auswertung des Fragebogens wurde das Statistikprogramm SPSS 14.0 verwendet.

\section{Gütekriterien}

Im Forschungsablauf wurden die sechs Gütekriterien aus allgemeinen Überlegungen der qualitativen Forschung: «Verfahrensdokumentation», «Regelgeleitetheit», «Argumentative Interpretationsabsicherung», «Nähe zum Gegenstand», «Kommunikative Validierung» berücksichtigt und ein triangulativer Ansatz angestrebt (Flick, 2006; Mayring, 2002). Da die Erstautorin selbst als Erfasserin in verschiedenen Beobachtungen mit der OERS involviert war, konnte insbesondere die Nähe zum Gegenstand gewährleistet werden.

\section{Ethische Richtlinien}

Das Gesamtprojekt ILQ, innerhalb welchem diese Untersuchung stattgefunden hatte, wurde im Jahr 2006 von der Kantonalen Ethikkommission Zürich bewilligt. Weil die vorliegende Studie mit gesunden Probandinnen durchgeführt worden war, die nicht dem $\mathrm{Pa}$ tientinnen- und Patientengesetz des Kantons Zürich (Gesundheitsdirek-

Tabelle 1: Beschreibung der Probandinnen.

\begin{tabular}{|lll|}
\hline Geschlecht & & Gruppe \\
\hline w & Bibliothekarin & A/Frau E \\
w & kaufmännische Ausbildung & A/Frau A \\
w & Fachfrau Gerontologie & A/Frau K \\
w & dipl. Gerontologin SAG, Berufsschullehrerin & A/Frau J \\
w & dipl. Pflegefachfrau, Fachfrau Gerontologie & B/Frau H \\
w & kaufmännische Ausbildung & B/Frau K \\
w & Fachfrau Gerontologie & B/Frau A \\
w & medizinische Laborantin & B/Frau M \\
w & Buchhalterin & C/Frau K \\
w & Fachfrau Gerontologie & C/Frau R \\
w & Pflegehelferin & C/Frau S \\
\hline$n=12$ & dipl. Gerontologin SAG & C/Frau M \\
\hline
\end{tabular}

tion, 2005) unterliegen, war eine neuerliche Bewilligung durch die Ethikkommission nicht erforderlich. Dem Recht auf Selbstbestimmung wurde durch eine informierte Zustimmung entsprochen, die sowohl mündlich als auch schriftlich erfolgt war.

\section{Ergebnisse}

\section{Stichprobe}

Es konnten aus der Gesamtpopulation $(N=29)$ im Pilotprojekt «Agitation» eine Stichprobe von 12 Probandinnen $(n=12)$ für die Teilnahme an den Fokusgruppeninterviews gewonnen werden, die in den Erfassungen mit der OERS in drei Langzeitinstitutionen im Kanton Zürich involviert waren. Das Alter der Probandinnen lag zwischen 47 und 80 Jahren, mit einem Durchschnittsalter von 60 Jahren. Sieben der zwölf Probandinnen (58\%) verfügten über eine pflegerische Qualifikation und damit auch über Erfahrungen in der Pflege und Betreuung von demenzerkrankten Personen. Aufgrund der geringen Anzahl der Probandinnen wurde von Einschluss- bzw. Aus- 
schlusskriterien abgesehen, was einer Gefälligkeitsstichprobe entsprach.

\section{Kategorienbildung}

Durch die Zusammenfassung wesentlicher Kernaussagen aus den Interviews konnten aus den Kodiereinheiten Schwerpunkte herausgearbeitet werden, aus denen sich sechs relevante Kategorien identifizieren ließen: «fehlende Eindeutigkeit», «fehlende Kodiermöglichkeiten», «Demenzschwere», «Einschätzen von Emotionen», «Einflussfaktoren» und «Anwenderschulung». Die Intercoder-Reliabilität wurde insofern berücksichtigt, als dass die Inhaltsklassifikation der Kodierungen von den Autorinnen im Wechsel auf Stabilität geprüft worden waren. Da der Schwerpunkt auf dem Erleben der Einschätzung von Emotionen mittels der OERS lag, findet die Kategorie «Anwenderschulung» im Rahmen der vorliegenden Untersuchung nur geringfügig Erwähnung. Die Angaben der jeweiligen Prozentzahlen beziehen sich auf die Anzahl der Befragten.

\section{Kategorie "fehlende Eindeutigkeit"}

Die Emotionen waren für die Probandinnen nicht immer eindeutig $\mathrm{zu}-$ zuordnen. Insbesondere «Alltagsgesichter», die wenig oder keine Mimik zeigten, erschwerten die Einschätzung der Emotionen mit der OERS. Auch beim wiederholten Beobachten von demenzerkrankten Bewohnern zeigten sich häufig die gleichen Emotionen, die nicht eindeutig zugeordnet werden konnten:

«Eine Person zeigte beim Essen immer das gleiche Gesicht von Traurigkeit, auch bei der Mahlzeit. Daher ist es schwer für mich gewesen, die Emotion als Traurigkeit einzuschätzen. Man konnte im vorgegebenen Rahmen die verschiedenen Nuancen nicht erfassen.» (Gruppe C, Frau M, 223-234)

Erschwert wurde die Einschätzung mit dem Instrument OERS, wenn die de- menzerkrankten Personen Gesichtszüge zeigten, die durch das Leben gezeichnet waren:

«Es hat Gesichter gegeben, die vom Leben so geprägt waren, dass die Mundwinkel hingen. Die Menschen waren aber deswegen nicht traurig oder ärgerlich.» (Gruppe B, Frau A, 93 - 99)

Emotionen wurden durch Müdigkeit überschattet, was das Einschätzen von Emotionen ebenso erschwerte, wie eine reduzierte Mimik, was Unsicherheit bei den Probandinnen auslöste:

«Es sind so viele Zwischentöne in den Emotionen, vor allem Müdigkeit. Die Zeit reicht manchmal einfach nicht aus, um zu definieren, was es ist, daher war ich unsicher.» (Gruppe A, Frau A, 41-55)

Die Ergebnisse aus den Fragebögen zeigten, dass die zwölf Probandinnen mehrheitlich der Meinung waren, dass das Einschätzen durch die fehlende Eindeutigkeit der Emotionen schwergefallen ist. Dass die sogenannten «Alltagsgesichter» die Einschätzung von Emotionen erschwerten, wurde mehrheitlich bestätigt (58,7\%). Auch die fehlende Eindeutigkeit, besonders zwischen Trauer und Ängstlichkeit, konnte von den Probandinnen mehrheitlich bestätigt werden $(83,3 \%)$.

Die Einschätzung von Emotionen ist den Probandinnen mehrheitlich leichtgefallen, wenn Emotionen auf den Gesichtern der demenzerkrankten Personen gut ablesbar waren (91,7\%). Die Erfasserinnen wünschten sich differenzierte Angaben zur Einschätzung von Emotionen. Demnach bestätigten sie, dass das Instrument zu wenig über die Qualität der Emotionen aussagt (57,4\%), was auch als fehlende Eindeutigkeit der Emotionen verstanden werden kann. Einheitlich wurde bestätigt, dass mit dem Instrument OERS nur zum Teil die Emotionen erfasst werden konnten, weil es an Eindeutigkeit fehlte $(100 \%)$. Mehrheitlich wurde bestätigt, dass es schwer war, Emotionen mit eher «traurigen» Gesichtszügen $(83,4 \%)$ Einzuschätzen. Ebenfalls bestätigten die Probandinnen, dass es zu wenige Auswahlmöglichkeiten zur Einschätzung von Emotionen gegeben hatte $(91,6 \%)$.

\section{Kategorie "fehlende Kodier- möglichkeiten"}

Fehlende Kodiermöglichkeiten lösten in der Bewertung Unzufriedenheit aus. Es entstand das Gefühl, den Beobachtungen nicht gerecht geworden zu sein; besonders, weil das Instrument es nicht vorsah, auch Informationen zur Gesamtsituation zu beschreiben. So äußerten die Probandinnen, dass ihnen Möglichkeiten gefehlt hatten, Angaben über die Beobachtungssituation zu machen, mit denen sie beobachtete Emotionen besser hätten erläutern können:

«Ganz viele Aspekte konnten mit dem Instrument gar nicht zum Ausdruck gebracht werden und wenn etwas passierte, ganz wenig, dann hat es einen anderen Ausdruck in der Mimik gegeben. Es hat mich gestört, dass ich dazu keine Angaben notieren konnte.» (Gruppe A, Frau K, 249-254)

Die Probandinnen hatten Schwierigkeiten, die Emotionen mit dem vorgegebenen Raster voneinander abzugrenzen. Besonders schwer war es häufig in Situationen, in denen demenzerkrankte Personen zufrieden wirkten:

«Beim Eintragen der Emotionen dachte ich manchmal, dass es schwierig sei, innerhalb der fünf Kategorien Emotionen zuzuordnen. Wenn jemand nur so dasitzt, ganz friedlich, dann habe ich nicht gewusst, welcher Zustand da inne ist. Ich habe bei Zufriedenheit mehr Gewicht auf Freude gelegt, aber mit einem schlechten Gewissen.» (Gruppe B, Frau M, 65-67)

Zugleich fehlten Kategorien zur differenzierten Einschätzung von Unruhe. Wurden sich wiederholende Bewegungen beobachtet, wussten die Pro- 
bandinnen vielmals nicht, wie sie die Beobachtung kodieren sollten:

«Ich fand es schwierig, repetive, agierte Bewegungen unter Ängstlichkeit zuzuordnen. Ich habe mich gefragt, ist das Ausdruck von Ängstlichkeit oder ist es Gewöhnung und Stimulation? Es fehlten im Instrument Kategorien für das ständige Brummen oder wenn jemand anfängt, mit den Füßen zu klopfen, sich kratzt oder an den Haaren herummacht.» (Gruppe A, Frau K, 729-734) Die Ergebnisse im Fragebogen fielen sehr heterogen aus. Repetiertes Verhalten wurde nicht einheitlich als Ängstlichkeit kodiert (50\%). Ebenso waren nicht alle Erfasserinnen unsicher bei der Einschätzung, wenn demenzerkrankte Personen beobachtet wurden, die längere Zeit an einem Ort gesessen hatten (50\%). Die Probandinnen gaben an, Zufriedenheit beobachtet zu haben, diese wurde aber nicht grundsätzlich als Freude kodiert (50\%). Wenn Bewohner Verhaltensweisen zeigten, die sich wiederholten, wurden diese nicht ignoriert (24,9\%). Somit kann aus diesen Ergebnissen interpretiert werden, dass ein Großteil der Probandinnen die OERS als nicht differenziert genug für eine adäquate Einschätzung beurteilt hatte.

\section{Kategorie "Demenzschwere"}

Aus allen Interviews wurde deutlich, dass das Stadium der Erkrankung einen wesentlichen Einfluss auf das Erkennenlassen von Emotionen zeigte. Häufig waren in der Beobachtung keine oder kaum Emotionen sichtbar:

«Ich konnte Emotionen nicht immer gleich gut erfassen, abhängig vom Schweregrad der Erkrankung. Es gab oft keine Regungen mehr zu beobachten. Bei den Schwerstdementen war es besonders schwer, da gab es keine Unterschiede zwischen ereignislosen Zeit und Aktivierung.» (Gruppe C, Frau K, 548-552)

Auch die Tageszeit, in der beobachtet wurde, nahm Einfluss auf die Qualität der Emotionen. Müdigkeit war ein häufiger Faktor, welcher das Einschätzen von Emotionen erschwert hatte:

«Emotionen sind auch bei Schwerstdementen sichtbar, sie können aber von Müdigkeit überschattet sein. Dann haben sie eine unterschiedliche Intensität und die Qualität kann mit dem Instrument nicht erfasst werden.» (Gruppe A, Frau K, 54-71)

Auch Aktivitäten begünstigten, dass demenzerkrankte Personen ermüdeten, was zur Folge hatte, dass Emotionen weniger sichtbar wurden:

«Ich habe erlebt, dass Demenzkranke am Abend zum Teil lebhafter gewesen waren aber zum Teil auch sehr müde, vor allem, wenn es eine Aktivierung gegeben hat.» (Gruppe A, Frau K, 115 -120)

Der Fragebogen bestätigte, dass die Ausprägung der Emotionen abhängig vom Schweregrad der Demenzerkrankung nicht gleich gut zu erkennen war (75\%). Dass es bei Bewohnern im fortgeschrittenen Demenzstadium besonders schwierig war, Emotionen einzuschätzen, bestätigten $58,4 \%$. Fast einheitlich bestätigten die Probandinnen jedoch, dass auch bei Personen mit einer sehr fortgeschrittenen Demenzerkrankung Emotionen sichtbar waren $(91,7 \%)$.

Kategorie "Einschätzen von Emotionen" Die Probandinnen hatten teilweise das Gefühl, dass die Beobachtungszeit von fünf Minuten zu kurz war, um Einschätzungen differenziert vornehmen zu können:

«Der Auftrag, fünf Minuten zu beobachten, war nicht schwierig, aber es war manchmal nur eine Minute, die gefehlt hat und die es ausgemacht hätte. So sind wichtige Beobachtungen verloren gegangen, die nicht berücksichtigt werden konnten.» (Gruppe B, Frau M, 244-246)

Das Einschätzen der Emotionen gelang den Probandinnen unterschiedlich gut. Auffallend war es, dass die Emotionen
Freude und Zufriedenheit häufig sicherer von anderen Emotionen abgegrenzt werden konnten:

«Bei einer ereignislosen Zeit hat es wenige Emotionen gegeben, Schläfrigkeit lie $\beta$ sich schlecht von Traurigkeit abgrenzen. Das Kodieren von Aufmerksamkeit und Freude ist mir besonders gut gelungen.» (Gruppe B, Frau K, 78-90)

Die Einschätzung von Emotionen mithilfe der OERS stellte für einzelne Probandinnen eine Herausforderung dar. Zudem hatten die Probandinnen das Gefühl, mehr und vielfältiger gesehen zu haben als tatsächlich mit dem Instrument festgehalten werden konnte: «Ich habe irgendwo das Gefühl gehabt, ich werde dem nicht gerecht, ich konnte das was ich beobachtet habe, mit dem Instrument nicht erfassen.» (Gruppe B, Frau H, 35 -40)

«Es war schwierig für mich, nichts über den Zustand der Frau schreiben zu können. Ich war unsicher in meiner Bewertung, bin dann nach meinem Gefühl, meiner Wahrnehmung vorgegangen.» (Gruppe C, Frau M, 152-160)

Dass es schwer war, in einer Zeit von fünf Minuten die Einschätzung von Emotionen vorzunehmen, wurde von den Probandinnen nur zum Teil bestätigt $(41,7 \%)$.

Die Mehrheit der Probandinnen verifizierte mit dem Fragebogen, dass sich die Freude am einfachsten kodieren ließ (91,7\%). Dass sich die Probandinnen unsicher bei der Bewertung von Emotionen gefühlt hatten, weil sich Schläfrigkeit schlecht von Traurigkeit abgrenzen ließ, wurde mehrheitlich falsifiziert (41,7\%). Ein apathisches Verhalten wurde als Traurigkeit eingestuft (25\%).

Die Probandinnen bestätigten, dass Emotionen auch in einer ereignislosen Zeit sichtbar waren (75\%). Dass nicht alles, was gesehen wurde, mit dem Instrument OERS aufgenommen werden konnte, wurde mehrheitlich durch das 
Antwortverhalten der Probandinnen bestätigt (83,3\%). So hatte die Mehrheit der Probandinnen das Gefühl, mehr und vielfältigere Emotionen gesehen zu haben als mit dem Instrument OERS kodiert werden konnten $(82,7 \%)$.

\section{Kategorie "Einflussfaktoren"}

Den Probandinnen ist aufgefallen, dass sich gewisse Bewohner an sie erinnert hatten. Dabei wurden einige unsicher und zeigten dies in ihren Verhaltensweisen, andere sprachen es direkt an oder fragten das Personal:

«Beobachtete Personen reagierten unterschiedlich, wenn ich mich von ihnen zurückzog. Mal waren sie traurig oder enttäuscht oder auch mal böse. Eine Frau sagte, ich solle verschwinden, ein Herr fühlte sich beobachtet und wurde sehr unruhig, ich musste die Beobachtung unterbrechen.» (Gruppe A, Frau K, 459-463)

Mehrheitlich ist es den Probandinnen schwergefallen, Beobachtungen unbeobachtet durchzuführen und sich gezielt abzugrenzen, was wiederum bei den Beteiligten Emotionen ausgelöst hatte:

«Unsere Anwesenheit nahm Einfluss auf die Atmosphäre. Beim zweiten Mal ist mir aufgefallen, dass einige Bewohner uns wahrnahmen. Sie erkannten uns auch nach vier bis fünf Wochen. Sie redeten über uns, merkten, dass wir sie beobachten.» (Gruppe A, Frau E, 95-102)

Bei anderen Beobachtungen löste das Beobachtet werden Ärger aus. Zudem entwickelten die Probandinnen das Gefühl, die Bewohner zu kennen und demnach abschätzen zu können, welche Emotionen sich zeigen würden:

«Eine Bewohnerin hat eine Emotion aufgrund meines Vorgehens gezeigt. Sie reagierte böse und als ich später wieder in den Raum kam, sagte sie: 〈Die Frau mit der Tasche soll verschwinden, raus, raus!'. Bei bestimmten Bewohnern habe ich mich an die letzten Beobachtungen erinnert und ebenso eingeschätzt.» (Gruppe A, Frau K, 474-480)

Die Probandinnen stellten fest, dass Verhaltensweisen von Pflegenden oder Personen aus dem direkten Wohnumfeld von Bewohnern mit Demenz dazu beigetragen hatten, dass positive Emotionen wie Freude oder Aufmerksamkeit beobachtet werden konnten:

«In der ereignislosen Zeit, wenn Mitarbeiter des Hauses vorbeigingen und den Bewohnern einen Blick zugeworfen haben, zeigten sich Emotionen. Auch bei einer kleinen Berührung war es, als würde das Gesicht des eben gerade noch schläfrigen Menschen wie eine Blume aufblühen, wenn auch nur für Sekunden.» (Gruppe A, Frau J, 329-335)

Die Ergebnisse aus den Fragebogen bestätigen, dass es den Probandinnen schwergefallen ist, sich von Bewohnern abzugrenzen, wenn diese sie als Gesprächspartner angesehen haben (58,4\%). Dass die Bewohner auf die Probandinnen innerhalb der Beobachtung reagierten, wurde mit etwas mehr als die Hälfte der Antworten bestätigt (66,6\%). Nur ein Teil der Probandinnen $(41,7 \%)$ hatte nicht den Eindruck, dass sich diese beobachtet gefühlt hatten. Für die Mehrheit der Probandinnen war jede Beobachtungssituation neu, so dass sie nicht im Voraus wussten, wie sich die Situation gestaltet (66,7\%).

\section{Kategorie "Anwenderschulung"}

Die Ergebnisse aus dem Fragebogen bestätigten, dass das Üben der Einschätzungen anhand der Filmsequenzen hilfreich war, um Emotionen mit der OERS einschätzen zu können. Einige Probandinnen hätten sich mehr Übungssequenzen gewünscht, um mehr Sicherheit zu erlangen.

«Die Schulung hätte an verschiedenen Stellen intensiver sein können, ich hätte mir mehr Übung mit dem Instrument gewünscht.» (Gruppe B, Frau A, $748-750)$

\section{Diskussion}

Verschiedene Untersuchungen zeigten, dass sich die OERS dazu eignet, Emotionen valide und reliabel zu erfassen (Lawton et al. 1996a; Snyder, Ryden, Shaver, Wang, Savik, Gross et al., 1998 ; Kolanowski, Litaker, Catalano, 2002). Allerdings wurde ebenfalls festgestellt, dass es mit dem Fortschreiten der Erkrankung oft auch schwieriger wird, Emotionen einzuschätzen, weil eine Verarmung des mimischen Ausdrucks stattfinden kann, welche mit der OERS nicht mehr festgehalten werden kann, weil diese in erster Linie versucht, durch abgebildete Gesichter mimische Ausdrucksmuster festzuhalten. Auch Becker, Kruse, Schröder und Seidl (2005) haben sich mit der Analyse mimischer Ausdrucksmuster beschäftigt und festgestellt, dass sich die OERS als reliables und valides Instrument zur Erfassung der emotionalen Befindlichkeit von Menschen mit Demenz in stationären Einrichtungen eignet.

Das Ergebnis der vorliegenden Untersuchung zeigt, dass es den Erfasserinnen schwergefallen ist, eine sehr feine und wenig ausgeprägte Mimik bei Menschen mit Demenz in der direkten Beobachtungssituation zu erkennen und mittels OERS festzuhalten. Ein Grund dafür, dass Emotionen schwer bis gar nicht erfasst werden konnten, könnte darin liegen, dass die Probandinnen keine einheitlichen Voraussetzungen für die Beobachtung mitgebracht hatten und dass mangelnde Kenntnisse über die Demenzerkrankung die Einschätzung erschwert hatten.

Die Methode der unbeteiligten Beobachtung wurde von den demenzerkrankten Bewohnern teilweise bemerkt und hatte somit Einfluss auf die Ergebnisse. Dies kann daran gelegen haben, dass sich die Probandinnen nicht optimal auf die Beobachtungssituation eingestellt haben, respektive in der Schu- 
lung nicht genügend darauf vorbereitet worden waren (Scheffer, 2002). Zudem gilt zu berücksichtigen, dass die räumlichen Gegebenheiten in den drei untersuchten Institutionen baulich sehr unterschiedlich waren. In einigen Fällen wurde auf schmalen Korridoren oder in kleinen Aufenthaltszimmern beobachtet, welche es erschwerten, genügend Distanz zu den Beobachteten einzuhalten.

Nicht zu unterschätzen ist auch der Umstand, dass die meisten Probandinnen kaum Erfahrungen im Umgang mit wissenschaftlichen Instrumenten hatten. Dies kann teilweise erklären, dass Unsicherheiten beim Einschätzen von Emotionen entstanden sind.

Die Ergebnisse der Interviews und des Fragebogens zeigen, dass es den Probandinnen leichtgefallen war, Freude und Aufmerksamkeit zu erkennen und $\mathrm{zu}$ kodieren. Allerdings wurde häufig auch die Zufriedenheit als emotionaler Zustand wahrgenommen, die sich mithilfe der OERS nicht hatte kodieren lassen. Eine frühere Version der OERS zeigte eine sechste Kodiermöglichkeit, nämlich die der Zufriedenheit (Lawton et al., 1996a; Snyder et al., 1998). Snyder et al. (1998) beschreiben den Zustand der Zufriedenheit als Ruhe bzw. Stille (tranquility). In der revidierten Version, die später auch unter dem Namen «Apparent Affect Rating Scale» von Lawton, Van Haitsma, Perkinson \& Ruckdeschel (1999) publiziert wurde und welche Gegenstand der vorliegenden Studie war, wurde diese Kategorie entfernt, weil sie nicht genügend trennscharf war und nicht genügend Varianz erklärt hatte. Auch wurde die Beobachtungszeit ursprünglich mit fünf bis zehn Minuten angegeben, wobei die Werte der Interrater-Reliabilität exzellent ausgefallen waren (Lawton et al., 1996a; Snyder et al., 1998). Bei der Planung der Untersuchung wurde davon ausgegangen, dass es in fünf Minuten gelingt, eine Datensättigung zu er- halten. Die Ergebnisse weisen nun darauf hin, dass die vorgegebene Zeit von fünf Minuten scheinbar nicht dazu ausreichte - respektive bei den Erfasserinnen das Gefühl hinterließ - der Einschätzung nicht gerecht geworden zu sein. Dies kann daran gelegen haben, dass diese über zu wenig Erfahrung im Umgang mit dem Instrument OERS verfügt hatten und dass wenige Kenntnisse über das Krankheitsbild der Demenz vorhanden waren. Ein zusätzlicher Faktor kann jedoch auch die jeweilige Einstellung zum Instrument selbst gewesen sein. So wurde innerhalb der Interviews deutlich, dass es einerseits für die Probandinnen schwer war, verschiedene Emotionen voneinander $\mathrm{zu}$ unterscheiden, andererseits fehlte Einzelnen die Überzeugung, dass Emotionen innerhalb von fünf Minuten erfasst werden könnten. Die Untersuchungen von Kruse (2004) zeigen, dass bei fortgeschrittener Demenz zwar ein differenziertes Einschätzen der Emotionen mit der OERS möglich ist, dass aber Pflegekräfte vielfach den Ausdruck von Freude im Sinne von Wohlbefinden deuteten. Die Probandinnen verdeutlichten mit ihren Aussagen, dass die Atmosphäre im direkten Wohnumfeld der demenzerkrankten Bewohnern zu positiven Emotionen beitragen kann, was mit der OERS gemessen werden konnte. Nach Lawton et al. (1996a) reagierten Personen mit Demenz besonders auf Zuwendung und Ansprache. Reaktionen zeigten sich auch auf nonverbale Zuwendung, durch einen Blick oder durch Berührungen, was durch Aussagen der Probandinnen in der vorliegenden Untersuchung bestätigt werden konnte. Snyder et al. (1998) stellten fest, dass in Situationen, in denen Aktivitäten stattgefunden hatten, Emotionen klarer erfasst werden konnten, was im Rahmen der vorliegenden Untersuchung untermauert werden konnte. Zudem zeigte sich, dass den Probandinnen besonders bei Aktivitäten und während der persönlichen Zuwendung das Einschätzen der Emotionen leichtgefallen war, weil sich Emotionen in Form von Freude bei den Betroffenen gezeigt hatten.

Nach Rösler, Schwerdt und RentelnKruse (2005) kann die Aufmerksamkeit von Pflegenden oder Personen im direkten Umfeld dazu beitragen, dass aufgrund ungewöhnlicher verbaler und nonverbaler Äußerungen Ressourcen von Menschen mit Demenz erkannt und gezielt gefördert werden konnten. Andererseits scheint eine gezielte Kommunikation - die auch nonverbal erfolgen konnte - es erst möglich zu machen, Kontakt zu Menschen aufzunehmen. Denn die Qualität der Kommunikation und die Gestaltung der Umgebung und des Tagesablaufes können einen nicht zu unterschätzenden Einfluss auf das subjektive Wohlbefinden der demenzerkrankten Bewohner haben.

Eine Schwäche der vorliegenden Studie besteht darin, dass es nicht immer möglich war, das Gespräch ausschließlich auf die Fragestellungen zu lenken. Im Datenmaterial ließen sich viele Informationen zum Pilotprojekt «Agitation» herausfiltern. Mit einer mehrmaligen Sichtung des Datenmaterials wurde ein großer Teil jener Kodiereinheiten gestrichen, die im Hinblick auf die Fragestellung wenig relevant erschienen waren. Eine weitere Schwäche der Studie lässt sich in der Selbstselektion der Probandinnen finden. Es wurden keine Einschluss- bzw. Ausschlusskriterien für die Teilnahme der Studie formuliert, was zu einer heterogenen Zusammensetzung der Probandinnen geführt hatte.

\section{Schlussfolgerungen}

Die Untersuchung hat gezeigt, dass fehlende Erfahrungen der Probandinnen im Umgang mit Demenz, eine 
zu kurze Schulung im Umgang mit dem Messinstrument und geringe Kenntnisse in der wissenschaftlichen Arbeitsweise sich auf eine objektive Einschätzung der Emotionen von Menschen mit Demenz negativ auswirken können. So können zum Beispiel fehlende Kenntnisse Unsicherheit hervorrufen und das valide Einschätzen von Emotionen erschweren. Ebenso kann die nötige Distanz von Pflegenden eine objektive und differenzierte Einschätzung von Emotionen verhindern.

In einer Folgeuntersuchung wäre zu überdenken, die Beobachtungszeit zwischen fünf und zehn Minuten für geübte Erfasserinnen $\mathrm{zu}$ erweitern. Ebenso könnte die gezielte Integration in die Pflegeplanung untersucht werden, um den Einsatz mit dem Instrument OERS zum Gegenstand einer reflektierten Pflegepraxis zu machen. Dies setzt jedoch voraus, dass Anwenderinnen von dem Einsatz der OERS überzeugt sind.

\section{How do users experience the handling of the Observed Emotional Rating Scale?}

As barely any empirical data on the application of the Observed Emotion Rating Scale (OERS) on sufferers of dementia is available for German-speaking Europe, the pilot project titled "Agitation" aimed to see how those persons entering the data get along with the OERS instrument.

For this purpose, 12 test persons were persuaded to carry out observations with the OERS instrument in three care centres located in the Swiss Canton of Zurich. The test persons were predominantly holders of nursing qualifications and/or experienced in caring for patients suffering from dementia.
Data collection was undertaken on the basis of three focus group interviews which were evaluated via qualitative content analysis. The preliminary results gained in the focus group interviews were validated by the same people using a questionnaire. The categories were titled "Missing Clearness", "Missing Possibility to Encode", "Level of Dementia", "Estimation of Emotions", "Influencing Factors" and "User Training". These indicated that knowledge of dementia is just as important as skilled use of OERS.

\section{Literatur}

Becker, S.; Kruse, A.; Schröder, J.; Seidl U.: Das Heidelberger Instrument zur Erfassung von Lebensqualität bei Demenz (H.I.L.DE.). Dimensionen von Lebensqualität und deren Operationalisierung. Zeitschrift für Gerontologie und Geriatrie, 38, 2005, 2: 108-21.

Flick, U.: Qualitative Sozialforschung. Rowohlt, Reinbek bei Hamburg, 2006.

Gesundheitsdirektion: Meine Rechte und Pflichten. Information zum Spitalaufenthalt, Gesundheitsdirektion Kanton Zürich, 2005.

Gonzalez-Salvador, T.; Lyketsos, C. G.; Baker, A.; Hovanec, L.; Roques, C., Brandt, J., Steele, C.: Quality of life in dementia patients in long-term care. International Journal of Geriatric Psychiatry, 15 2000, 2: $181-189$.

Häder, M.: Empirische Sozialforschung. Eine Einführung. VS Verlag für Sozialwissenschaften, Wiesbaden, 2006.

Höpflinger, F.; Hugentobler, V.: Pflegebedürftigkeit in der Schweiz. Prognosen und Szenarien für das 21. Jahrhundert. Buchreihe des Ge- sundheitsobservatoriums. Verlag Hans Huber, Bern, 2004.

Kolanowski, A.; Litaker, M. S.; Catalano, P. A.: Emotional Well-Being in a Person with Dementia. Western Journal of Nursing Research, 24, 2002, 1: $28-48$.

Kean, S.: Focus Group Interviews: Ein qualitativer Forschungsansatz in der Pflege. Pflege, 2000, 13: $145-151$.

Kruse, A.: Gemeinsam für ein besseres Leben mit Demenz in Wissenschaft und Gesellschaft. Robert Bosch Stiftung, 2004.

Lamnek, S.; Qualitative Sozialforschung. Lehrbuch. Psychologie Verlag Union München, Weinheim, 2005.

Lawton, M. P.; Van Haitsma, K.; Klapper, $\mathrm{J} .:$ Observed affect in nursing home residents with Alzheimer's disease. Journal of Gerontology, Psychological Sciences, 51B, 1996a, 1: 3-14.

Lawton, M. P.; Van Haitsma, K.; Klapper, J.: Observed Emotion Rating Scale, 1996b. Internetressource. Abgerufen am 02.01.2009 unter http://www.abramsoncenter.org/ pri/documents/observedemotion. pdf.

Lawton, M. P.; Van Haitsma, K.; Perkinson, M.; Ruckdeschel, K.: Observed Affect and Quality of Life in Dementia: Further Affirmations and Problems. Journal of Mental Health and Aging, 5, 1999, 1: 69-81.

Mayring, P.: Einführung in die qualitative Sozialforschung. Beltz, Weinheim und Basel, 2002.

Mayring, P.: Qualitative Inhaltsanalyse. Grundlagen und Techniken. UTB, Stuttgart, 2003.

Oppikofer, S.: Zürcher Schriften zur Gerontologie.Pflegeinterventionen bei Agitation und schwerer Demenz. Ein Pilotprojekt zur Überprüfung der Praxistauglichkeit von Instrumenten zur Messung der Lebensqualität bei Menschen mit 
schwerer Demenz anhand einer Pflegeintervention. Universität Zürich, Zentrum für Gerontologie, 2008.

Rösler, A.; Schwerdt, R.; von RentelnKruse, W.: Was die Sprache Alzheimer-Kranker mit der Celans verbindet. Über Kommunikation mit schwer betroffenen Demenzpatienten. Zeitschrift für Gerontologie und Geriatrie, 38, 2005, 5: 354-359.

Scheffer, T.: Das Beobachten als sozialwissenschaftliche Methode - Von den Grenzen der Beobachtbarkeit und ihrer methodischen Bearbeitung. In: Schaeffer, D.; Müller-
Mundt, G.: Qualitative Gesundheits- und Pflegeforschung. Verlag Hans Huber, Bern, 2002.

Snyder, M.; Ryden, M. B.; Shaver, P.; Wang, J. J.; Savik, K.; Gross, C. R.; Pearson, V:: The Apparent Emotion Rating Instrument: Assessing Affect in Cognitively Impaired Elders. Clinical Gerontologist, 18, 1998, 4: $17-29$.

Philadelphia Geriatric Center: Recognizing and Responding To Emotion In Persons With Dementia. Terra Nova Films, Chicago, 1996. Internetressource. Abgerufen am 15.12.2008 unter: http://www.terranova.org/
Weyerer, S.: Altersdemenz. In: Gesundheitsberichtserstattung des Bundes, Statistisches Bundesamt. 28, RobertKoch-Institut, Berlin, 2005.

\section{Korrespondenzadresse}

Mag. Daniela Händler-Schuster Dipl.-Berufspäd. (FH)

ZAG Zentrum für Ausbildung im Gesundheitswesen Kanton Zürich Turbinenstrasse 5

CH-8400 Winterthur

E-Mail: daniela.haendler@zag.zh.ch 